\title{
Location of lacunar infarcts correlates with cognition in a sample of non-disabled subjects with age-related white-matter changes: the LADIS study
}

\author{
S Benisty, ${ }^{1}$ A A Gouw, ${ }^{2}$ R Porcher, ${ }^{3}$ S Madureira, ${ }^{4} \mathrm{~K}$ Hernandez, ${ }^{1}$ A Poggesi, ${ }^{5}$ W M van \\ der Flier, ${ }^{2}$ E C W Van Straaten, ${ }^{2}$ A Verdelho, ${ }^{4} \mathrm{~J}$ Ferro, ${ }^{4} \mathrm{~L}$ Pantoni, ${ }^{5} \mathrm{D}$ Inzitari, ${ }^{5} \mathrm{~F}$ Barkhof, ${ }^{2}$ \\ F Fazekas, ${ }^{6} \mathrm{H}$ Chabriat ${ }^{1}$, on behalf of the LADIS Study group
}

\begin{abstract}
${ }^{1}$ Departments of Neurology and Geriatrics, Lariboisière-Fernand Widal Hospital, APHP, Paris, France; ${ }^{2}$ Departments of Neurology and Radiology, VU Medical Center, Amsterdam, The Netherlands; ${ }^{3}$ Department of Biostatistics and Medical Informatics, Saint Louis Hospital, APHP and University Paris Diderot, Paris, France; ${ }^{4}$ Serviço de Neurologia, Centro de Estudos Egas Moniz, Hospital de Santa Maria, Lisbon, Portugal;

${ }^{5}$ Department of Neurological and Psychiatric Sciences, University of Florence, Florence, Italy; ${ }^{6}$ Department of Neurology and MRI Institute, Karl-Franzens University Graz, Graz, Austria
\end{abstract}

Correspondence to:

Dr H Chabriat, Department of Neurology, Lariboisiere-Fernand Widal Hospital, APHP, University Paris 7, 2 rue Ambroise Paré, 75010 Paris, France; hugues.chabriat@lrb.aphp.fr

Received 19 August 2008 Revised 10 December 2008 Accepted 11 December 2008 Published Online First 11 February 2009

\section{ABSTRACT}

Objectives: In cerebral small vessel disease, whitematter hyperintensities (WMH) and lacunes are both related to cognition. Still, their respective contribution in older people remains unclear. The purpose of this study is to assess the topographic distribution of lacunes and determine whether it has an impact on cognitive functions in a sample of non-disabled patients with age-related white-matter changes.

Methods: Data were drawn from the baseline evaluation of the LADIS (Leucoaraioisis and Disability study) cohort of non-disabled subjects beyond 65 years of age. The neuropsychological evaluation was based on the Mini Mental Status Examination (MMSE), a modified Alzheimer Diseases Assessment Scale for global cognitive functions, and compound $Z$ scores for memory, executive functions, speed and motor control. WMH were rated according to the Fazekas scale; the number of lacunes was assessed in the following areas: lobar white matter, putamen/ pallidum, thalamus, caudate nucleus, internal/external capsule, infratentorial areas. An analysis of covariance was performed after adjustment for possible confounders. Results: Among 633 subjects, 47\% had at least one lacune ( $31 \%$ at least one within basal ganglia). The presence of lacunes in the thalamus was associated with lower scores of MMSE ( $\beta=-0.61 ; p=0.043)$, and worse compound scores for speed and motor control $(\beta=-0.25 ; p=0.006)$, executive functions $(\beta=-0.19 ; p=0.022)$ independently of the cognitive impact of WMH. There was also a significant negative association between the presence of lacunes in putamen/ pallidum and the memory compound $Z$ score $(\beta=-0.13$; $p=0.038)$. By contrast, no significant negative association was found between cognitive parameters and the presence of lacunes in internal capsule, lobar white matter and caudate nucleus.

Conclusion: In non-disabled elderly subjects with leucoaraisosis, the location of lacunes within subcortical grey matter is a determinant of cognitive impairment, independently of the extent of $\mathrm{WMH}$.

Ischaemic cerebral small vessel disease is characterised by two main types of lesions on brain MRI, white-matter hyperintensities (WMH) and lacunar infarcts. ${ }^{1}$ This entity is frequent in elderly people and has been related to cognitive impairment in demented patients, as well as in non-disabled elderly. ${ }^{2}$ Still, the relative contribution of WMH and lacunar infarcts to cognitive dysfunction, particularly in these populations, has not been clarified. Most previous clinicoradiological studies indicated a significant association between the extent of WMH and both cognitive impairment and decline in older people. ${ }^{3-6}$ However, the potential influence of associated lacunar infarcts was rarely investigated. In addition, some results cast doubts on an independent impact of lacunar infarcts on cognition in older people. Finally, the main determinants of the cognitive impact of cerebral lacunar infarcts, particularly the exact role of their number and/or location, remain poorly studied in large populations of elderly subjects.

The Leucoaraiosis and Disability (LADIS) study is a prospective multinational European study investigating the impact of $\mathrm{WMH}$ in the transition to disability of a cohort of non-disabled elderly subjects with varying degrees of small-vessel disease. $^{8}$ Using data obtained at baseline, we previously reported that lacunar infarcts and WMH were independently associated with global cognitive performances in this population. ${ }^{9}$ In the present study, we investigated whether the location of lacunes in different subcortical structures has a significant and independent influence on cognitive performances in the same sample. In particular, the location of lacunar infarcts in various subcortical structures was analysed, since some of these structures, such as the thalamus, have been reported as locations for strategic infarcts in vascular dementia ${ }^{10}$ and are involved in cognition. ${ }^{11} 12$

\section{MATERIALS AND METHODS}

Data were drawn from the multicentre, multinational Leucoaraiosis and Disability (LADIS) study. In short, 639 elderly subjects, who had no or only mild disability in their Instrumental Activities of Daily Living (IADL) and showed WMH, were enrolled. Subjects presented for evaluation in the following settings: stroke units or stroke departments, cerebrovascular disease clinics, memory or dementia clinics, neurological or geriatric wards/clinics, population studies on ageing and controls in other studies. Among the reasons for presentation were mild memory loss, minor motor disturbances, minor focal cerebrovascular events or non-specific reasons for undergoing a cranial neuroimaging study (WMH as incidental finding). To be included, subjects had to (1) be of an age between 65 and 84 years; (2) have WMH on MRI of any degree, according to the classification into the three severity classes of the Fazekas scale; ${ }^{13}$ 
(3) have no or only mild disability as determined by the IADL scale; ${ }^{14}$ (4) show evidence of a regularly contactable informant; and (5) agree to sign an informed consent. Exclusion criteria were (1) likely drop out because of the presence of severe illnesses (cardiac, hepatic or renal failure, cancer or other relevant systemic diseases), (2) severe unrelated neurological diseases, (3) leucoencephalopathy of non-vascular origin (immunological demyelinating, metabolic, toxic, infectious, other), (4) severe psychiatric disorders, (5) inability to give informed consent and (6) inability or refusal to undergo cerebral MRI. Subjects have been followed up for 3 years with repeated clinical and MRI studies. The present study is based on the baseline data of the LADIS project.

\section{Neuropsychological evaluation}

The neuropsychological battery used in the LADIS study is detailed elsewhere. ${ }^{15}$

General cognitive function was measured using the Mini Mental Status Examination $(\mathrm{MMSE})^{16}$ and a modified version of the Alzheimer Diseases Assessment Scale (ADAS Mod). ${ }^{17}$ The ADAS was slightly modified to include the item "delayed word recall." A higher MMSE indicated better performances, whereas a higher ADAS Mod score corresponded to worse performances.

Performances by cognitive domains were compared using $Z$ standard scores to calculate compound measures. These compound measures were based on subtests of ADAS Mod and on the Stroop ${ }^{18} 19$ and Trail making test. ${ }^{20}$ Three main domains were analysed: (1) compound Z score for memory domain $=Z$-scores of (Immediate word recall+delayed recall+word recognition+digit span)/4; (2) compound $Z$ score for executive functions domain $=Z$-scores of (Stroop test+Trail making+Symbol digit+Verbal fluency)/4; (3) compound $Z$ scores for speed and motor control domain $=$ Z-scores of (Trail making part A+Mazes+Digit cancellation)/3. Z scores of tests with higher scores representing worse performance were inverted $(-Z)$ in order to calculate these compound measure scores. Memory, executive functions and speed and motor control compound Z-scores were respectively missing for one, 53 and 13 subjects. Therefore, for these three variables, the statistical analysis was performed with a different number of subjects.

\section{MRI procedure}

All subjects were studied by MRI following a standard protocol on the same day as the clinical investigation. Scans were made on a $0.5 \mathrm{~T}$ (one centre) or $1.5 \mathrm{~T}$ scanner (10 centres) and were collected at the Image Analysis Center of the Vrije Universiteit Medical Center, Amsterdam. Imaging guidelines were distributed among all centres, specifying name convention and scan positioning. To check and maintain quality, dummy runs were requested before the beginning of the study and in case of upgrades. Data transfer was done using a CD-ROM or through FTP. The MR protocol included the following sequences: T1weighed 3D MPRAGE (magnetisation prepared rapid-acquisition gradient-echo, scan parameters: coronal or sagittal plane, field of view (FOV) $250 \mathrm{~mm}$, matrix $256 \times 256$ or $512 \times 512$, slice thickness: $1 \mathrm{~mm}$ (isotopic voxels),; TE: 2-7 ms; TR: 9-26 ms, FA 10-30\%), T2-weighed FSE (fast spin echo, scan parameters: axial plane, FOV $250 \mathrm{~mm}$, matrix $256 \times 256$ or $512 \times 512$, slice thickness: $5 \mathrm{~mm}$, interslice gap $0.5 \mathrm{~mm}$, TE: $100-130 \mathrm{~ms}$, TR: 4000-8000 ms), and FLAIR (Fluid Attenuated Inversion Recovery; scan parameters: axial plane, FOV $250 \mathrm{~mm}$; matrix $256 \times 256$ or $512 \times 512$; slice thickness: $5 \mathrm{~mm}$, interslice gap
$0.5 \mathrm{~mm}$; TE: 100 to $160 \mathrm{~ms}$; TR: 6000-10 $000 \mathrm{~ms}$; TI: $2000-$ 2400).

Visual ratings of WMH and lacunes were performed by raters who were blind to clinical details. The degree of WMH severity was rated visually by a single rater (ECWvS) on axial FLAIR images using the Fazekas Scale as grade 1 (punctate), grade 2 (early confluent) or grade 3 (confluent). The intrarater reliability of rating WMH was determined on 18 randomly selected scans that were scored twice (weighted Cohen $\kappa=0.84$ ). Lacunes were defined as hypointense foci of $\geqslant 3 \mathrm{~mm}$ on MPRAGE that were surrounded by white matter or subcortical grey matter and not located in areas of high prevalence of widened perivascular spaces (eg, anterior commissure, vertex). The number of lacunes was recoded into none, few (one to three lacunes) and many (four lacunes or more). In patients with lacunes, their number was first assessed in the following structures on both sides: lobar white matter, basal ganglia (including thalamus), infratentorial areas. ${ }^{9}$ In patients with lacunes present within the basal ganglia, the number and location of lacunes were assessed on both sides and after consensus by two raters ( $\mathrm{SB}, \mathrm{AP})$ in the following structures: putamen, pallidum, caudate nucleus, internal and external capsule, and thalamus. The inter-rater and intrarater reliabilities of this procedure were not determined. During the evaluation of follow-up MRI scans, baseline MRI rating was corrected in 22 scans. This correction was necessary because of a mistake in the data processing. The correction proved not to affect the main results of the baseline analysis but has resulted in small differences in some data reported in the present paper compared with those reported in previous publications.

\section{Statistical analysis}

The association between lacune location and each of the outcomes was assessed using Analysis of Covariance adjusted for age, sex, education, diabetes, hypertension, alcohol consumption, history of stroke, depression and for grades of WMH according to the Fazekas scale. Additionally, the analyses were adjusted for the centre of origin, treated as a random effect in the model. For each outcome, separate models were first fitted for the presence of lacunes in each of the predetermined locations. In order to reduce the number of parameters tested in the model, we chose to estimate at most four different regression coefficients. For this purpose, we considered only locations with coefficients greater than $20 \%$ of their standard deviation. Thereafter, we set identical effect for locations with nearly equal coefficients as judged by comparing their difference to the corresponding standard error. A differential effect related to the location of lacunes was then assessed by a test of heterogeneity of the coefficients. The association between each location and the outcome was tested only in the presence of a significant heterogeneity. Analyses evaluating the side effect were carried out using a framework similar to the main analysis and a model with different effects according to the side of lacunes. Such analyses were restricted to locations and endpoints where a significant effect was shown.

The models were fitted using restricted maximum likelihood (REML), as usually favoured. However, comparisons of models involving different fixed effects were based on maximum likelihood estimates (ML), as likelihood comparisons between REML fits with different fixed effects are not valid. The comparisons of nested models were carried out using likelihood ratio tests. The comparisons of non-nested models were based on Akaike's information criterion (AIC). 
Results are expressed in terms of estimated regression parameter ( $\beta$ ) and standard error (SE), as well as the Wald test for the parameter.

All tests were two-sided, at the 0.05 significance level. Analyses were performed using R 2.5.1 statistical software (The R Foundation for Statistical computing, Vienna, Austria).

\section{RESULTS}

In the LADIS cohort, 639 patients were included. Of these, six were excluded because the ADAS score was missing. Analysis was thus performed on a sample of 633 patients. Characteristics of the participants, including sociodemographic factors, risk factors and cognitive scores are presented in table 1.

Three hundred patients (47\% of the total population) presented with at least one cerebral lacunar infarct (114 of them had a history of stroke), and the total number of lacunar infarcts was 958. Among these patients, 64\% had at least one lacune within basal ganglia, $16 \%$ in caudate nucleus, $30 \%$ in putamen and pallidum, $12 \%$ in internal capsule, $12 \%$ in external capsule and $25 \%$ in thalamus. The topographic distribution of all lacunar infarcts and their range in each area is presented in table 2 .

The adjusted association between location and load of lacunes, WMH and general cognitive performances are presented in table 3.

The association between the presence of lacunes in any location and MMSE scores was found to be significant after adjustment for potential confounders $(\beta(\mathrm{SE})=-0.31(0.14)$, $\mathrm{p}=0.023)$.

When the regional location of lacunes was included in the analysis, a negative association with MMSE scores was found to be significant only when lacunes were located in the thalamus $(p=0.043)$. A significant differential effect related to the location of lacunes was detected (test of heterogeneity: $p=0.033$ ). In particular, the impact of the presence of lacunes in the thalamus on the MMSE was found to be three times greater than the effect of the presence of lacunes in the caudate and twice the effect of the presence of lacunes in other locations. In contrast, a trend for a positive association was

Table 1 Characteristics of the 633 patients

\begin{tabular}{lc}
\hline Age, years (SD) & $74(5)$ \\
Female gender (\%) & $350(55)$ \\
Education, years (SD) & $10(4)$ \\
Hypertension (\%) & $439(70)$ \\
Diabetes (\%) & $91(15)$ \\
Alcohol consumption & \\
Yes (\%) & $301(48)$ \\
Yes, in the past (\%) & $48(8)$ \\
No (\%) & $282(45)$ \\
Depressive symptoms (\%) & $176(28)$ \\
History of stroke (\%) & $180(29)$ \\
Mini Mental State Examination (SD) & $27(2)$ \\
Alzheimer Disease Assessment Scale (SD) & $17(7)$ \\
White-matter hyperintensities & \\
Mild (\%) & $281(44)$ \\
Moderate (\%) & $196(31)$ \\
Severe (\%) & $156(25)$ \\
Lacunes & \\
None (0) (\%) & $333(53)$ \\
Few (1 to 3) (\%) & $217(34)$ \\
Many (4 or more) (\%) & $83(13)$ \\
Cortical infarct (\%) & $57(9)$ \\
\hline
\end{tabular}

Values are mean (SD) or frequency (\%). detected between MMSE and the presence of lacunes in the internal capsule, which suggests a relative preservation of MMSE scores in the presence of lesions in this location. Finally, the model with lacune locations had a better AIC than the model with the number of lacunes irrespective of their location, despite three more degrees of freedom (2765.2 vs 2762.8). No side effect of lacunes in the thalamus was detected on MMSE $(p=0.58)$, speed and motor compound $Z$ score $(p=0.31)$ and executive functions compound $Z$ score $(p=0.74)$. Nor was any side effect detected for lesions in putamen and pallidum on memory compound Z score $(p=0.75)$.

The association between the presence of lacunes in any location with the ADAS mod scores did not reach statistical significance. The only significant association detected with lacunar infarcts located in the caudate suggested that the corresponding performances were relatively preserved in the presence of lesions at this location.

Finally, a significant association was observed between the extent of WMH and both MMSE and ADAS Mod.

The adjusted associations between location and load of lacunes, WMH and compound $\mathrm{Z}$ scores are presented in table 4.

A significant association was found between the number of lacunes (none/few/many) and the compound $Z$ score for executive functions but not with the compound $Z$ scores for memory or for speed and motor control.

The heterogeneity test of the different locations of lacunes was significant for all compound $Z$ scores. For the memory compound $Z$ score, a significant effect of the presence of lacunes in the putamen and/or pallidum was detected $(p=0.038)$. For the speed and motor control, a significant association was detected for the presence of lacunes in the thalamus $(p=0.006)$ with a lower effect of the presence of lacunes in the putamen, pallidum, external capsule and infratentorial areas $(p=0.049$ and $p=0.047)$. Finally, for executive functions, a differential effect of the presence of lacunes in the thalamus $(p=0.022)$, in the lobar white-matter $(p=0.048)$ or in the putamen and pallidum (although non-significant, $(p=0.010)$ ) was observed.

A significant association was observed between the extent of WMH and all the compound Z scores.

\section{DISCUSSION}

The main result of this study is that, in non-disabled elderly subjects with leucoaraiosis, the cognitive impact of lacunar infarcts depends on their location and not only on their total number. According to these data, the location of lesions in three structures, mainly the thalamus, but also the putamen and pallidum, may be involved in cognitive performances. In the same cohort, we previously observed an independent impact of both white-matter lesions and lacunar infarcts on cognition, after correction for multiple confounders. ${ }^{9}$ In the present study, after adjustment for WMH severity, we showed that the presence of lacunar infarcts within some specific subcortical structures is also significantly and independently associated with worse cognitive performances.

These results are in line with former postmortem data ${ }^{21}$ showing that lacunes in the thalamus and basal ganglia significantly predicted cognitive status and were a better predictor of the cognitive status than periventricular or diffuse "leucoaraïosis." 21 Conversely, although Mungas et al observed that the volume of lacunes in the thalamus was associated with several cognitive performances in elderly subjects, their results were no longer significant when the extent of white-matter lesions was also considered in their model. Besides, the results of Mungas et al were obtained in a widely heterogeneous sample of 
Table 2 Topographic distribution of all lacunar infarcts and their range in each area

\begin{tabular}{lcl}
\hline & N (\%) present & $\begin{array}{l}\text { Mean (range) no of } \\
\text { lacunes when present }\end{array}$ \\
\hline Any location & $300(47 \%)$ & $3.2(1$ to 25$)$ \\
Lobar white matter & $171(27 \%)$ & $2.4(1$ to 25$)$ \\
$\quad$ Frontal & $129(20 \%)$ & $2.0(1$ to 19$)$ \\
Parieto-occipital & $70(11 \%)$ & $1.5(1$ to 4$)$ \\
Temporal & $32(5 \%)$ & $1.4(1$ to 5$)$ \\
Infratentorial & $76(12 \%)$ & $1.5(1$ to 8$)$ \\
Basal ganglia & $193(31 \%)$ & $2.2(1$ to 15$)$ \\
Caudate nucleus & $49(8 \%)$ & $1.3(1$ to 3$)$ \\
Putamen and pallidum & $107(17 \%)$ & $1.4(1$ to 4$)$ \\
Internal capsule & $35(5 \%)$ & $1.2(1$ to 2$)$ \\
Thalamus & $74(12 \%)$ & $1.4(1$ to 6$)$ \\
External capsule & $37(6 \%)$ & $1.6(1$ to 4$)$ \\
\hline
\end{tabular}

Patient number $(n)$ of and percentage of the total 633 patients $(\%)$.

both demented and non-demented subjects. In such a sample, the weight of hippocampal atrophy and/or diffuse brain damage may have blurred a more limited impact of lacunar lesions on cognitive parameters. In the Rotterdam study, a prospective population-based cohort designed to study causes and consequences of brain changes in older people, Vermeer et al observed that the presence of silent infarcts (mainly lacunar infarcts) was also associated with worse performances at baseline using a global cognitive compound score, but they did not find a significant decline in MMSE score after an average follow-up of 3.6 years. Interestingly, they detected a significant decline in memory performances when lacunar infarcts were located in the thalamus at baseline and a larger decline in psychomotor speed when these lesions were located elsewhere, ${ }^{22}$ suggestive of a differential impact of location of these lesions as observed in the present study.

In this study, the presence of lacunes in the thalamus was associated with worse performances in global cognitive function as well as in both speed and motor control and executive functions. Noteworthily, the impact on global cognitive function was only observed with the MMSE, though this scale was only mildly impaired in non-disabled subjects. By contrast, no significant association was detected with the ADAS Mod score except for lacunar infarcts located in the caudate nucleus suggesting that the corresponding performances were relatively preserved in the presence of lesions at this location. This may be due to the fact that this scale is adapted from the ADAS cog, designed for detecting the progression of Alzheimer disease, especially at moderate stages, ${ }^{23}$ and is not a good tool for detecting mild cognitive changes. ${ }^{24}$ Besides, when specific cognitive domains were considered in the analysis, speed and motor control were affected by the presence of lacunar infarcts in the thalamus. This result is in line with previous data showing that (1) speed of information processing is frequently impaired in patients with thalamic lesions ${ }^{12}$ and (2) age-related decrease in thalamic volume is associated with reduced speed processing. ${ }^{25}$ In addition, in the present study, the location of lacunar infarcts in the thalamus was associated with a lower compound $\mathrm{Z}$ score for executive functions in line with previous reports in thalamic infarctions. ${ }^{12}$ In contrast, no significant association was detected between the thalamic location and memory performances. In 22 cases with thalamic infarction, Van der Werf et al reported that an amnestic syndrome was not necessarily related to lesions involving only the mamillothalamic tract. ${ }^{12}$ Beside, simple attention deficit was found to be a rather general trait of thalamic lesions and did not appear associated with a specific structure within the thalamus. Thus, in our population, the thalamic lesions may not have been severe enough or properly localised to alter significantly memory performances. Alternatively, the use of compound scores for the assessment of different cognitive domains instead of single tests as well as the lack of detailed anatomical analysis of lacunar thalamic infarcts may also have reduced our ability to detect some discrete effects on memory functions. In this study, a significant effect of location of lesions in the putamen or pallidum was detected on memory performances. This is in line with persisting short-term memory impairment already reported after the occurrence of unilateral lenticular infarcts. ${ }^{26}$ However, in contrast with thalamic infarcts, no significant effect was detected on MMSE scores. Finally, the strategic role of lesions located in the internal capsule on cognition ${ }^{27}$ was not observed in this study but cannot be excluded in the absence of detailed location analysis.

Our study also sheds light on the relative contribution of lacunar infarcts (number and location) and WMH on cognition. Interestingly, the load of lacunar infarcts was found to be associated only with the compound Z score for executive functions, whereas WMH had a significant effect on all general cognitive and compound scores. In contrast with the absence of impact of their number, the location of lacunar infarcts within

Table 3 Adjusted association between location and load of lacunes, white-matter hyperintensities and general cognitive performance

\begin{tabular}{|c|c|c|c|c|}
\hline & \multicolumn{2}{|c|}{ Mini Mental Status Examination } & \multicolumn{2}{|c|}{ Alzheimer Disease Assessment Scale } \\
\hline & $\beta$ (SE) & p Value & $\beta$ (SE) & p Value \\
\hline $\begin{array}{l}\text { White-matter hyperintensities } \\
\text { Lacunes }\end{array}$ & $-0.32(0.12)$ & 0.007 & $1.07(0.34)$ & 0.002 \\
\hline Any location (none/few/many) & $-0.31(0.14)$ & 0.023 & $0.20(0.39)$ & 0.61 \\
\hline Presence in each location & & & & \\
\hline Thalamus & $-0.61(0.30)^{1}$ & 0.043 & $-0.04(0.86)$ & 0.96 \\
\hline Caudate & $-0.17(0.35)^{2}$ & 0.63 & $-2.06(1.01)$ & 0.042 \\
\hline Internal capsula & $0.77(0.40)^{3}$ & 0.051 & $-0.83(1.14)$ & 0.47 \\
\hline Putamen and pallidum & $-0.02(0.26)$ & 0.95 & $1.08(0.75)$ & 0.15 \\
\hline Lobar white matter & $-0.30(0.22)^{4}$ & 0.17 & $0.47(0.64)$ & 0.46 \\
\hline External capsula and infratentorial & $-0.31(0.25)^{4}$ & 0.22 & $0.89(0.73)$ & 0.23 \\
\hline Heterogeneity of effect & Between 1, 2, 3 and 4 & 0.033 & - & - \\
\hline
\end{tabular}

Bold values denote significant associations with altered cognitive performance $(p<0.05)$. Bold and italic values signify an opposite effect suggestive of relatively preserved performance. The heterogeneity effect was tested between four subgroups of merged coefficients, as indicated by superscripts. 
Table 4 Adjusted associations between location and load of lacunes, white-matter hyperintensities and compound Z scores

\begin{tabular}{|c|c|c|c|c|c|c|}
\hline & \multicolumn{2}{|l|}{ Memory (Z score) } & \multicolumn{2}{|c|}{ Speed and motor control (Z score) } & \multicolumn{2}{|c|}{ Executive functions (Z score) } \\
\hline & $\beta$ (SE) & p Value & $\beta$ (SE) & p Value & $\beta$ (SE) & p Value \\
\hline $\begin{array}{l}\text { White-matter hyperintensities } \\
\text { Lacunes }\end{array}$ & $-0.08(0.03)$ & 0.014 & $-013(0.04)$ & 0.0006 & $-0.14(0.10)$ & $<0.0001$ \\
\hline Any location (none/few/many) & $-0.01(0.04)$ & 0.74 & $-0.06(0.04)$ & 0.15 & $-0.08(0.04)$ & 0.039 \\
\hline \multicolumn{7}{|l|}{ Presence in each location } \\
\hline Thalamus & $-0.09(0.08)^{1}$ & 0.26 & $-0.25(0.09)^{1}$ & 0.006 & $-0.19(0.08)^{1}$ & 0.022 \\
\hline Caudate & $0.13(0.09)^{2}$ & 0.13 & $-0.05(0.10)^{2}$ & 0.65 & $0.10(0.10)^{3}$ & 0.31 \\
\hline Putamen and pallidum & $-0.13(0.06)^{3}$ & 0.038 & $-0.15(0.08)^{3}$ & 0.049 & $-0.11(0.07)^{2}$ & 0.10 \\
\hline Lobar white matter & $0.0003(0.06)$ & $>0.99$ & $-0.02(0.07)^{2}$ & 0.82 & $-0.12(0.06)^{2}$ & 0.048 \\
\hline External capsula and infratentorial & $-0.03(0.07)^{1}$ & 0.66 & $-0.15(0.08)^{3}$ & 0.047 & $-0.03(0.07)^{3}$ & 0.73 \\
\hline Heterogeneity effect & Between 1, 2, 3 & 0.005 & Between 1, 2, 3 & 0.02 & Between 1, 2, 3 & 0.036 \\
\hline
\end{tabular}

Bold values denote significant associations with altered cognitive performance $(p<0.05)$. The heterogeneity effect was tested between three subgroups of merged coefficients as indicated by superscripts.

the thalamus was found to be associated with speed and motor control. Moreover, the importance of location of lacunar infarcts as a determinant of cognitive impairment is also supported by the following results: (1) there was a significant heterogeneity effect of lacunes location on cognitive performances, (2) a model with separate effects for the presence of lacunar infarcts in the thalamus and in other regions was found to be better suited than one based only on the number of infarcts to fit the relationships between MMSE and MRI lesions.

The strengths of this study are the large number of elderly patients and the relative homogeneity of the cognitive status of the population made of non-demented and non-disabled subjects with leucoaraiosis. The limitations are the analysis of cross-sectional data with the need of confirmation in a prospective aspect, the different number of patients for analysis of the three composite $Z$ scores due to a different number of missing data, the lack of very detailed anatomical analysis and the use of compound measures of cognitive performances which might have hampered the detection of subtle cognitive effects of infarcts location. Analysing the effect of lacune location was also a challenging task because this effect partly combines with the potential influence of the number of lacunes. In the present study, we considered that lacune location would be related to cognitive impairment if the coefficients obtained for different locations were significantly different in a multiple regression model (analysis of covariance). This corresponds to comparing a regression model with equal coefficients whatever the location, to a model with separate coefficients for each location. In the LADIS sample, the number of patients with lacunes in each location was limited. Consequently, the study has a limited ability to detect a differential effect according to lacune location. To overcome this difficulty, we preprocessed the data in order to diminish the number of parameters estimated and to decrease the variance of parameter estimates. This is a potential limitation of the study as well as the difficulty in estimating the exact effect of lacune location and that of lacune number. We showed that for MMSE, the model with lacune locations better fitted the data than a model with the number of lacunes irrespective of their location. However, the respective impact of number and location could not be fully evaluated and would deserve a larger study. In future studies, it will also be useful to take into consideration cerebral atrophy, whose impact on cognition has been recently recognised in small-vessel disease. ${ }^{28}$ Finally, the findings in this population may not be valid at more severe stages of small-vessel disease or in demented subjects, particularly in the presence of large amounts of lacunar infarcts and/or severe cerebral atrophy whose effects could exceed that of subcortical location of ischaemic lesions.

In conclusion, our study emphasised the impact of WMH on memory, speed and motor control, and executive functions. Moreover, it showed that not only the load but also the location of "silent" lacunar infarcts, especially in specific subcortical structures, are additional determinants of cognitive impairment in non-disabled elderly subjects. Whether the location of lacunar lesions increases the risk of cognitive decline and disability in this population will require further investigations and prospective studies.

Funding: The LADIS study is supported by the European Union within the V European Framework Program "Ouality of life and management of living resources" (19982002), contract no QLRT-2000-00446 as a concerted action.

\section{Competing interests: None.}

Ethics approval: Ethics approval was provided by the institutions mentioned in the Appendix.

Patient consent: Obtained.

\section{REFERENCES}

1. O'Brien JT, Erkinjuntti T, Reisberg B, et al. Vascular cognitive impairment. Lancet Neurol 2003;2:89-98.

2. Roman GC, Erkinjuntti T, Wallin A, et al. Subcortical ischaemic vascular dementia Lancet Neurol 2002;1:426-36.

3. Longstreth WT, Manolio TA, Arnold A, et al. Clinical correlates of white matter findings on cranial magnetic resonance imaging of 3301 elderly people: The Cardiovascular Health Study. Stroke 1996;27:1274-82.

4. de Groot JC, de Leeuw FE, Oudkerk M, et al. Cerebral white matter lesions and cognitive function: the Rotterdam Scan Study. Ann Neurol 2000;47:145-51.

5. De Groot JC, De Leeuw FE, Oudkerk M, et al. Periventricular cerebral white matter lesions predict rate of cognitive decline. Ann Neurol 2002;52:335-41.

6. Garde E, Mortensen EL, Krabbe K, et al. Relation between age-related decline in intelligence and cerebral white-matter hyperintensities in healthy octogenarians: a longitudinal study. Lancet 2000;356:628-34.

7. Mungas D, Jagust WJ, Reed BR, et al. MRI predictors of cognition in subcortical ischemic vascular disease and Alzheimer's disease. Neurology 2001;57:2229-35.

8. Pantoni L, Basile AM, Pracucci G, et al. Impact of age-related cerebral white matter changes on the transition to disability - the LADIS study: rationale, design and methodology. Neuroepidemiology 2005;24:51-62.

9. van der Flier WM, van Straaten EC, Barkhof F, et al. Small vessel disease and general cognitive function in nondisabled elderly: the LADIS study. Stroke 2005;36:2116-20.

10. Szirmai I, Vastagh I, Szombathelyi E, et al. Strategic infarcts of the thalamus in vascular dementia. J Neurol Sci 2002;203-204:91-7.

11. Exner C, Weniger G, Irle E. Implicit and explicit memory after focal thalamic lesions. Neurology 2001;57:2054-63.

12. Van der Werf YD, Scheltens P, Lindeboom J, et al. Deficits of memory, executive functioning and attention following infarction in the thalamus; a study of 22 cases with localised lesions. Neuropsychologia 2003;41:1330-44.

13. Fazekas F, Chawluk JB, Alavi A, et al. MR signal abnormalities at $1.5 \mathrm{~T}$ in Alzheimer's dementia and normal aging. Am J Roentgenol 1987;149:351-6. 
14. Lawton M, Brody E. Assessment of older people: self maintaining and instrumental activities of daily living. Gerontologist 1969;9:179-86.

15. Madureira S, Verdelho A, Ferro J, et al. Development of a neuropsychological battery for the Leukoaraiosis and Disability in the Elderly Study (LADIS): experience and baseline data. Neuroepidemiology 2006;27:101-16

16. Folstein M, Folstein S, McHugh P. Mini-mental state. A practical method for grading the cognitive state of patients for the clinician. J Psychiatr Res 1975:189-98.

17. Rosen WG, Mohs RC, Davis KL. A new rating scale for Alzheimer's disease. Am J Psychiatry 1984;141:1356-64.

18. Stroop J. Studies of interference in serial verbal reactions. J Exp Psychol 1935; 18:643-62

19. McLeod C. Half a century of research on the Stroop effect: an integrative review. Psychol Bull 1991;109:163-203.

20. Reitan RM. The relation of the trail making test to organic brain damage. J Consult Psychol 1955;19:393-4.

21. Gold G, Kovari E, Herrmann FR, et al. Cognitive consequences of thalamic, basal ganglia, and deep white matter lacunes in brain aging and dementia. Stroke 2005;36:1184-8.

22. Vermeer SE, Prins ND, den Heijer T, et al. Silent brain infarcts and the risk of dementia and cognitive decline. N Engl J Med 2003;348:1215-22.

23. Doraiswamy PM, Kaiser L, Bieber F, et al. The Alzheimer's Disease Assessment Scale: evaluation of psychometric properties and patterns of cognitive decline in multicenter clinical trials of mild to moderate Alzheimer's disease. Alzheimer Dis Assoc Disord 2001;15:174-83.

24. Ylikoski R, Jokinen $\mathrm{H}$, Andersen $\mathrm{P}$, et al. Comparison of the Alzheimer's Disease Assessment Scale Cognitive Subscale and the Vascular Dementia Assessment Scale in differentiating elderly individuals with different degrees of white matter changes. The LADIS Study. Dement Geriatr Cogn Disord 2007;24:73-81.

25. Van Der Werf YD, Tisserand DJ, Visser PJ, et al. Thalamic volume predicts performance on tests of cognitive speed and decreases in healthy aging: A magnetic resonance imaging-based volumetric analysis. Cogn Brain Res 2001;11:377-85.

26. Giroud M, Lemesle M, Madinier G, et al. Unilateral lenticular infarcts: radiological and clinical syndromes, aetiology, and prognosis. J Neurol Neurosurg Psychiatry 1997;63:611-15

27. Tatemichi TK, Desmond DW, Prohovnik I, et al. Confusion and memory loss from capsular genu infarction: a thalamocortical disconnection syndrome? Neurology 1992;42:1966-79.
28. Jouvent $\mathbf{E}$, Viswanathan A, Mangin JF, et al. Brain atrophy is related to lacunar lesions and tissue microstructural changes in CADASIL. Stroke 2007;38:1786-90.

\section{APPENDIX. LIST OF PARTICIPATING CENTRES AND PERSONNEL}

Helsinki, Finland (Memory Research Unit, Department of Clinical Neurosciences, Helsinki University): T Erkinjuntti, T Pohjasvaara, P Pihanen, R Ylikoski, H Jokinen, M-M Somerkoski, R Mäntylä, O Salonen; Graz, Austria (Department of Neurology and Department of Radiology, Division of Neuroradiology, Medical University Graz): F Fazekas, R Schmidt, S Ropele, B Rous, K Petrovic, U Garmehi, A Seewann; Lisboa, Portugal (Serviço de Neurologia, Centro de Estudos Egas Moniz, Hospital de Santa Maria): JM Ferro, A Verdelho, S Madureira, C Moleiro; Amsterdam, The Netherlands (Department of Radiology and Neurology, VU Medical Center): P Scheltens, I van Straaten, F Barkhof, A Gouw, W van der Flier; Goteborg, Sweden (Institute of Clinical Neuroscience, Goteborg University): A Wallin, M Jonsson, K Lind, A Nordlund, S Rolstad, I Isblad; Huddinge, Sweden (Karolinska Institutet, Department of Neurobiology, Care Sciences and Society; Karolinska University Hospital Huddinge): L-O Wahlund, M Crisby, A Pettersson, K Amberla; Paris, France (Department of Neurology, Hopital Lariboisiere): H Chabriat, K Hernandez, A Kurtz, D Hervé, S Benisty, JP Guichard; Mannheim, Germany (Department of Neurology, University of Heidelberg Klinikum Mannheim): M Hennerici, C Blahak, H Baezner, M Wiarda, S Seip; Copenhagen, Denmark (Memory Disorders Research Group, Department of Neurology, Rigshospitalet, and the Danish Research Center for Magnetic Resonance, Hvidovre Hospital, Copenhagen University Hospitals): G Waldemar, E Rostrup; C Ryberg, T Dyrby, OB Paulson; E Garde; Newcastle upon Tyne, UK (Institute for Ageing and Health, Newcastle University): J O'Brien, S Pakrasi, M Krishnan, A Teodorczuk, M Firbank, P English, T Minett. The coordinating centre is in Florence, Italy (Department of Neurological and Psychiatric Sciences, University of Florence): D Inzitari (Study Coordinator); L Bartolini, AM Basile, E Magnani, M Martini, M Mascalchi, M Moretti, L Pantoni, A Poggesi, G Pracucci, E Salvadori, M Simoni. The LADIS Steering Committee is formed by D Inzitari (study coordinator), T Erkinjuntti, P Scheltens, M Visser and P Langhorne, who replaced in this role K Asplund beginning with 2005.

\section{Stay a step ahead with Online First}

We publish all our original articles online before they appear in a print issue. This means that the latest clinical research papers go straight from acceptance to your browser, keeping you at the cutting edge of medicine. We update the site weekly so that it remains as topical as possible. Follow the Online First link on the home page and read the latest research. 